\title{
CYLINDRIC ALGEBRAS OF FIRST-ORDER LANGUAGES
}

\author{
BY \\ DALE MYERS( $\left.{ }^{1}\right)$
}

\begin{abstract}
We show when two countable first-order languages have isomorphic cylindric algebras.
\end{abstract}

Introduction. The cylindric algebra of a language is the $\omega$-dimensional cylindric algebra of formulas of the language modulo logical equivalence. We classify first-order languages according to the isomorphism types of their cylindric algebras. For two languages involving only predicate symbols, their cylindric algebras are isomorphic iff for every $n \in \omega$, both languages have the same number of predicate symbols with at least $n$ places. This solves the classification problem for free $\omega$-dimensional locally-finite cylindric algebras [4, Problem 2.8, p. 463] since these algebras are exactly the cylindric algebras of languages involving only predicate symbols.

For any first-order theory, the topological space of its models with $E C_{\Delta}$ classes (classes which consist of all models of some theory) as closed sets is a natural dual for the Lindenbaum-Tarski algebra of the theory. By enriching the class of models to the concrete category of models and isomorphisms and replacing the topology with the ultraproduct operations, we obtain a natural model-theoretic dual for the cylindric algebra of formulas of the theory. This duality is used in demonstrating that various properties of languages are invariant under cylindric isomorphism.

The basic tool used in isomorphism construction is a decomposition version of the Cantor back-and-forth construction [7].

Notation. All languages, theories and sets of symbols will be countable. All theories will be first-order theories with equality. "Symbol" will mean nonlogical symbol. The type of an $n$-ary predicate symbol is $n$; that of an $n$-ary operation symbol is $n+1 / 2$. Propositional letters are regarded as 0 -ary predicate symbols and constants as 0 -ary operation symbols. Let $1 / 2 \omega=\left\{1 / 2,1 \frac{1}{2}, 21 / 2, \ldots\right\}$. The (full) type sequence of a language is the sequence $\left\langle a_{0}, a_{1 / 2}, a_{1}, a_{1 \frac{1}{2}}, \ldots\right\rangle$ where $a_{i}$ is the number (possibly $\omega$ ) of symbols of the language of type $i$. The reduced type sequence is the sequence $\left\langle b_{0}, b_{1 / 2}, b_{1}, b_{1 / 2}, \ldots\right\rangle$ where $b_{i}$ is $\omega$ if there are

Received by the editors September 23, 1974.

AMS (MOS) subject classifications (1970). Primary 02J15.

Key words and phrases. First-order languages, cylindric algebras, back-and-forth constructions, dual space of a cylindric algebra, category of models, ultraproducts.

$\left.{ }^{1}\right)$ This research was partially supported by NSF Grant GP-43327. 
infinitely many symbols of type $\geqslant i$ and, if not, the number of symbols of type $i$. The unboundedness of the language is the largest $i$, if any, such that $b_{i}=\omega$; it is undefined if no $b_{i}$ is $\omega$ and $\omega$ if every $b_{i}$ is $\omega$.

We shall occasionally wish to consider symbols other than equality as quasilogical built-in symbols. Let $S$ be a fixed set of such symbols, let $\Sigma$ be a set of axioms on $S$ and let $s=\langle S, \Sigma\rangle$. For any set $R$ of symbols, let $L_{s}(R)$ be the language whose set of symbols is $S \cup R$ and let $T h_{s}(R)$ be the theory whose language is $L_{s}(R)$ and whose axioms are $\Sigma$. We write $L(R)$ and $T h(R)$ for $L_{s}(R)$ and $T h_{s}(R)$ when $s=\langle\varnothing, \varnothing\rangle$. Suppose $T$ is a theory whose set of symbols includes $S$. If $R^{\prime}$ is a subset of $T$ 's set of symbols, let $T \mid R^{\prime}$ be the restriction of $T$ to the sentences of $L_{s}\left(R^{\prime}\right)$. If $\sigma$ is a sentence of $T$ 's language, let $T \sigma$ be the theory obtained by adding $\sigma$ to the axioms of $T$. Let $\operatorname{cyl}_{s}(T)$ be the cylindric algebra of formula's of $T$ 's language modulo equivalence with respect to $T$ plus constants $a_{S}$ for $S \in S$ where $a_{S}$ is $S x_{1} \ldots x_{n}$ if $S$ is an $n$-ary predicate symbol and $S x_{1}$ $\cdots x_{n}=x_{n+1}$ if $S$ is an $n$-ary operation symbol. Let $\cong_{s}$ be the relation on theories such that $T \cong{ }_{s} T^{\prime}$ iff $\operatorname{cyl}_{s}(T) \cong \operatorname{cyl}_{s}\left(T^{\prime}\right)$. We write $\cong$ for $\cong_{s}$ when $s=$ $\langle\varnothing, \varnothing\rangle$. If $T \cong T^{\prime}, T$ and $T^{\prime}$ are cylindrically isomorphic. Being cylindrically isomorphic is equivalent to being bilaterally interpretable [6], being synonymous [1] or, if there are no constant or operation symbols, being related [8].

Cylindric duality. In this section and the next, the $S$ and $\Sigma$ of the previous section will be empty. Let Cyl be the category consisting of the algebras $\operatorname{cyl}(T)$, $T$ a theory, and cylindric homomorphisms. Given a theory $T$, an ultraproduct operation on the class of its models is one which, for some index set $I$ and ultrafilter $D$, sends an $I$-indexed family $\left\langle\mathscr{Q}_{i}: i \in I\right\rangle$ of models of $T$ to the ultraproduct $\Pi_{D} \mathfrak{U}_{i}$. Let mod-ult $(T)$ be the concrete category of models of $T$ and isomorphisms operated on by the ultraproduct operations. Let Mod-Ult be the category whose objects are the categories mod-ult $(T), T$ a theory, and whose morphisms are functors $h: \bmod -u l t(T) \rightarrow$ mod-ult $\left(T^{\prime}\right)$ such that $h \circ$ (the forgetful functor) $=$ the identity and $h$ commutes with all ultraproduct operations.

TheOREM 1 (DuAlty THEOREM). The categories Cyl and Mod-Ult are dual.

Proof. For any theory $T$, let $\operatorname{cyl}(T)^{*}=\bmod -u l t(T)$. Let $T$ and $T^{\prime}$ be theories and suppose for convenience that their symbols are the binary predicates $R$ and $S$ respectively. For any $f: \operatorname{cyl}\left(T^{\prime}\right) \rightarrow \operatorname{cyl}(T)$, let $f^{*}: \bmod -u l t(T) \rightarrow$ $\bmod$-ult $\left(T^{\prime}\right)$ be the unique concrete functor such that for any model $\mathfrak{U}=$ $\left\langle X, R^{\mathfrak{U}}\right\rangle$ of $T, f^{*}\left(\left\langle X, R^{\mathfrak{U}}\right\rangle\right)=\left\langle X, \varphi^{\mathfrak{I}}\right\rangle$ where $\varphi(x, y)=f(S x y)$ and $\varphi^{\mathfrak{I}}$ is the interpretation of $\varphi$ in $\mathcal{U}$. The structure $\left\langle X, \varphi^{\mathfrak{U}}\right\rangle$ is a model of $T^{\prime}$ since if $T^{\prime} \vDash$ $\sigma$ then, since $f$ is a cylindric homomorphism, $T=f(\sigma)$ and hence $\left\langle X, R^{\mathscr{\ell}}\right\rangle \vDash$ $f(\sigma)$ and hence, since $f(\sigma)$ is the result of replacing $S$ with $\varphi$ in $\sigma,\left\langle X, \varphi^{\mathfrak{l}}\right\rangle \vDash \sigma$. Clearly, $f^{*}$ preserves ultraproducts. 
Easily, if $i$ is an identity map of Cyl, then $i^{*}$ is an identity map of ModUlt and if $f$ and $g$ are maps of Cyl, then $(f \circ g)^{*}=g^{*} \circ f^{*}$. Hence ()$^{*}$ is a contravariant functor from Cyl to Mod-Ult.

For any theory $T$ let mod-ult $(T)^{*}=\operatorname{cyl}(T)$. Again suppose $T$ and $T^{\prime}$ are theories whose symbols are the binary predicates $R$ and $S$ respectively. Let $h$ : mod-ult $(T) \rightarrow$ mod-ult $\left(T^{\prime}\right)$ be a functor in Mod-Ult. Then the map $U_{h}$ defined by $U_{h}(\mathscr{U})=\left\langle X, R^{\mathfrak{\imath}}, S^{h(\mathfrak{U})}\right\rangle$ for $\mathscr{\imath}=\left\langle X, R^{\mathfrak{\imath l}}\right\rangle$ commutes with the ultraproduct operations and preserves isomorphisms. Hence by Kochen [6, Theorem 12.1] and Shelah [9] (the latter shows that the ultralimit condition of the former may be omitted) there is a formula $\varphi(x, y)$ involving only $R$ such that for any $2=$ $\left\langle X, R^{\mathfrak{U}}\right\rangle, \varphi^{\mathfrak{U}}=S^{h(\mathfrak{U})}$. Clearly this $\varphi(x, y)$ is unique modulo equivalence with repsect to $T$. Let $f: \operatorname{cyl}(L(\{S\})) \rightarrow \operatorname{cyl}(T)$ be the unique homomorphism such that $f(S x y)=\varphi(x, y)$ and let $p: \operatorname{cyl}(L(\{S\})) \rightarrow \operatorname{cyl}\left(T^{\prime}\right)$ be the homomorphism such that $p(S x y)=S x y$. For any sentence $\sigma$ of $L(\{S\})$, if $T^{\prime} \vDash \sigma$ and $\mathscr{\imath} \vDash T$ then $h(\mathfrak{l}) \vDash T^{\prime}$ and so $h(\mathfrak{Q}) \vDash \sigma$ and so $\mathfrak{Q} \vDash f(\sigma)$. Hence $T^{\prime} \vDash \sigma$ implies $T \vDash$ $f(\sigma)$ and so $f$ factors through $p$ and, since $p$ is onto, the factorization is unique. Let $h^{*}$ be the unique homomorphism such that $f=h^{*} \circ p$. Easily, for any identity map $i$ of Mod-Ult, $i{ }^{*}$ is an idertity map of Cyl and for any $h$ and $g,(h \circ g)^{*}=g^{*} \circ h^{*}$. Finally it is clear that $\operatorname{cyl}(T)^{* *}=\operatorname{cyl}(T)$ and $\bmod -u l t(T)^{* *}=\bmod$-ult $(T)$. It is also straightforward to verify that for any map $h$ in Mod-Ult, $h^{* *}=h$. For any map $f$ in Cyl, $f^{* *}=f$ follows from the uniqueness modulo equivalence with respect to $T$ of the $\varphi(x, y)$ in the definition above.

COROLlary 2. For any theories $T$ and $T^{\prime}$, if $\operatorname{cyl}(T) \cong \operatorname{cyl}\left(T^{\prime}\right)$, then

(1) the number of models of $T$ with a given universe equals the number of models of $T^{\prime}$ with that universe,

(2) the number of nonisomorphic models of $T$ of a given cardinality equals the number of nonisomorphic models of $T^{\prime}$ of that cardinality, and

(3) every automorphism group of a model of $T$ is also (identical with not just isomorphic to) an automorphism group of a model of $T^{\prime}$ and vice versa.

Our model-theoretic dual should be compared to the sheaf-theoretic duals of S. D. Comer [2] which are part model-theoretic (the base space is the topological space of models) and part algebraic (the stalks are cylindric algebras).

Invariants. An invariant of a language is a property of or a function on languages such that languages with isomorphic cylindric algebras are the same with respect to the property or are assigned the same value by the function.

Although the full type sequence is not an invariant, we have

LEMma 3. The reduced type sequence is an invariant.

Proof. The proof is basically the counting argument of Pigozzi in [4, 
Theorem 2.5.51]. We show that languages with different reduced type sequences are not cylindrically isomorphic. Let $T$ and $T^{\prime}$ be the theories of languages $L$ and $L^{\prime}$ whose sets of symbols are $R$ and $R^{\prime}$ and whose reduced type sequences are $\alpha=$ $\left\langle a_{0}, a_{1 / 2}, a_{1}, \ldots\right\rangle$ and $\beta=\left\langle b_{0}, b_{1 / 2}, b_{1}, \ldots\right\rangle$. If $\alpha \neq \beta$, then, since they are reduced type sequences, one of $\alpha$ and $\beta$ must be smaller reverse lexicographically. Suppose for some $i, a_{i}>b_{i}$ and $a_{j} \geqslant b_{j}$ for $j>i$. Thus $b_{i}<\omega$ and so $b_{j}<\omega$ for $j>i$ and for some $k \geqslant i, b_{j}=0$ for $j \geqslant k$. Suppose for reductio ad absurdum that $f: \operatorname{cyl}(T) \cong \operatorname{cyl}\left(T^{\prime}\right)$. Let $R_{0}$ be a subset of $R$ consisting of $b_{i}+1$ symbols of type $i$ and $b_{j}$ symbols of type $j$ for $j \in(i, k]$. Then $f$ maps cyl $\left(T \mid R_{0}\right) 1-1$ into $\operatorname{cyl}\left(T^{\prime} \mid R_{0}^{\prime}\right)$ for some finite $R_{0}^{\prime} \subseteq R^{\prime}$ consisting of $b_{i}^{\prime}$ symbols of type $i$ for $i \in$ $[0, k]$ where $b_{i}^{\prime} \leqslant b_{i}$. Hence $f^{*}$, the dual of $f$ defined in the Duality section, is a universe preserving map from the structures of $L\left(R_{0}^{\prime}\right)$ to those of $L\left(R_{0}\right)$. If 2 is a structure of $L\left(R_{0}\right)$ with finite universe and $\sigma$ a sentence of $\operatorname{Cyl}\left(T \mid R_{0}\right)$ whose model class is 2 's isomorphism type, then 2 not in the image of $f^{*}$ implies $f(\sigma)=$ 0 which is impossible since $f$ is $1-1$. Hence $f^{*}$ maps the finite structures of $L\left(R_{0}^{\prime}\right)$ onto the finite structures of $L\left(R_{0}\right)$. Let $x$ be a finite cardinal and let $N_{0}(x)$ and $N_{0}^{\prime}(x)$ be the number of $L\left(R_{0}\right)$-structures and $L\left(R_{0}^{\prime}\right)$-structures, respectively, over a given universe of cardinality $x$. Thus $N_{0}(x) \leqslant N_{0}^{\prime}(x)$. For illustrative purposes, suppose $i \in \omega$ and $k \in 1 / 2 \omega$. Then

$$
\begin{aligned}
& N_{0}(x)=\left(2^{\left(x^{i}\right)}\right)^{b_{i}+1}\left(x^{\left(x^{i}\right)}\right)^{b_{i+1 / 2}}\left(2^{\left(x^{i+1}\right)}\right)^{b_{i+1}} \cdots\left(x^{\left(x^{k-1 / 2}\right)}\right)^{b_{k}}, \quad \text { and } \\
& N_{0}^{\prime}(x)=\left(2^{\left(x^{0}\right)}\right)^{b_{0}^{\prime}}\left(x^{\left(x^{0}\right)}\right)^{b_{1 / 2}^{\prime}} \cdots\left(2^{\left(x^{i}\right)}\right)^{b_{i}^{\prime}} \cdots\left(x^{\left(x^{k-1 / 2}\right)}\right)^{b_{k}^{\prime}}
\end{aligned}
$$

Taking logarithms we have

$$
\log _{2} N_{0}(x)=\left(b_{i}+1\right) x^{i}+b_{i+1 / 2} x^{i} \log _{2} x+b_{i+1} x^{i+1}+\cdots+b_{k} x^{k-1 / 2} \log _{2} x,
$$

and

$$
\log _{2} N_{0}^{\prime}(x)=b_{0}^{\prime} x^{0}+b_{1 / 2}^{\prime} x^{0} \log _{2} x+\cdots+b_{i}^{\prime} x^{i}+\cdots+b_{k}^{\prime} x^{k-1 / 2} \log _{2} x .
$$

Since $b_{i}+1>b_{i}^{\prime}$ and $b_{j} \geqslant b_{j}^{\prime}$ for $j \in(i, k]$, a simple rate of growth argument shows that $\log _{2} N_{0}(x)>\log _{2} N_{0}^{\prime}(x)$ for sufficiently large $x$. But this contradicts $N_{0}(x) \leqslant N_{0}^{\prime}(x)$. Hence $T$ and $T^{\prime}$ are not cylindrically isomorphic.

LEMMA 4. The existence of a constant is an invariant.

Proof. Any structure of a language with a constant has a point which is left fixed by all automorphsims. Any language without a constant has a structure with no such fixed point. Hence no language with a constant is cylindrically isomorphic to one without a constant.

LEMMA 5. The number of predicate symbols is an invariant. 
Proof. The number of isomorphism types of one element structures of a language with $m$ predicate symbols is $2^{m}$ and, by Corollary 2 , this number is an invariant.

LEMMA 6. The number of unary predicate symbols is an invariant for the class of languages whose symbols are of type 0,1 or $1 \frac{112}{2}$.

Proof. Suppose $L$ and $L^{\prime}$ are languages all of whose symbols are of type 0 , 1 or $1 \frac{1}{2}$; suppose $L$ has $k$ unary predicate symbols, $k \in \omega \cup\{\omega\}$; and suppose $L^{\prime}$ has $k^{\prime}$ unary predicate symbols, $k^{\prime}<k$. By interpreting the function symbols to be the identity and by choosing appropriate interpretations for the unary predicates, we can construct an $L$-structure with an infinite universe $X$ which can be partitioned into $2^{k}$ infinite orbits such that the restriction of the automorphism group of the structure to an orbit is the full symmetric group of that orbit. In any $L^{\prime}$-structure over $X$ with the same automorphism group all the function symbols must be interpreted as identities since if $f(x) \neq x$ we can find an automorphism which moves $f(x)$ but not $x$, an impossibility. But any such $L^{\prime}$-structure can be partitioned into at most $2^{k^{\prime}}$ orbits. Hence $k^{\prime}<k$ is impossible.

Isomorphism construction. Given an index set $\mathbf{S}$, let $\mathbf{C}$ be the category of countable $\omega$-dimensional locally-finite cylindric algebras with an $S$-indexed list of constants and homomorphisms which preserve the constants. The minimal subalgebra of an algebra in $\mathbf{C}$ is the subalgebra generated by the constants. Let $\oplus$ : $\mathbf{C} \times \mathbf{C} \rightarrow \mathbf{C}$ be the cartesian product functor and let $\otimes: \mathbf{C} \times \mathbf{C} \rightarrow \mathbf{C}$ be the tensor product functor. For objects $\mathscr{U}$ and $\mathbb{B}$ of $C, \mathscr{U} \otimes \mathbb{B}$ is the categorical direct sum of $\mathscr{U}$ and $B$ in $C$. If there are no additional constants, $\mathscr{U} \otimes B$ is the free product of the cylindric algebras $\mathfrak{U}$ and $\mathfrak{B}$. If there are additional constants and $\mathscr{O}$ and $\mathbb{B}$ have the same minimal algebra $\mathfrak{E}, \mathscr{Q} \otimes \mathbb{B}$ is the free product of $\mathscr{Q}$ and $\mathbb{P}$ amalgamated over $\sqrt{ }$ with constants interpreted as in $\sqrt{ }$. Let $\Phi$ be the class of all polynomials in $\otimes$ and $\oplus$ with coefficients in $\mathbf{C}$. This is the smallest class of functors closed under composition and containing $\otimes, \oplus$, the identity and projection functors, and all zero-ary functors whose unique value is in $\mathbf{C}$.

If $\supseteqq$ is the minimal subalgebra of $\mathscr{Q}, \supseteqq \rightarrow \mathscr{U}$ will always be the canonical injection. If maps $\mathfrak{N}_{1} \rightarrow \mathfrak{B}_{1}, \ldots, \mathfrak{I}_{n} \rightarrow \mathfrak{B}_{n}$ have been given, $\varphi\left(\mathscr{U}_{1}, \ldots, \mathfrak{Q}_{n}\right)$ $\rightarrow \varphi\left(\mathfrak{B}_{1}, \ldots, \mathfrak{B}_{n}\right)$ will always be the map $\varphi\left(\mathfrak{A}_{1} \rightarrow \mathfrak{B}_{1}, \ldots, \mathfrak{A}_{n} \rightarrow \mathfrak{B}_{n}\right)$.

A symmetric binary relation $\mathbf{Q}$ on the algebras of $\mathbf{C}$ is $\Phi$-decomposable iff for any algebras $\mathscr{U}$ and $B$ of $C$, (D1) $\mathcal{Q} B$ implies $\mathscr{A}$ and $\mathscr{B}$ have isomorphic minimal subalgebras and $(\mathrm{D} 2) \mathbb{Z} Q \mathbb{Q}$ and $\mathbb{S} \rightarrow$ IO a monomorphism from a finitely generated algebra of $\mathbf{C}$ implies there is a functor $\varphi\left(X_{1}, \ldots, X_{n}\right)$ in $\Phi$, sequences $\overline{\tilde{U}}=$ $\left\langle\mathscr{U}_{1}, \ldots, \mathscr{V}_{n}\right\rangle$ and $\bar{B}=\left\langle\mathbb{B}_{1}, \ldots, \mathbb{B}_{n}\right\rangle$ of algebras in $C$ and isomorphisms $\varphi\left(\mathscr{U}_{1}, \ldots, \mathscr{Q}_{n}\right) \cong \mathscr{U}$ and $\varphi\left(\mathfrak{B}_{1}, \ldots, \mathbb{B}_{n}\right) \cong \mathbb{B}$ such that $\mathscr{U}_{1} \mathrm{Q} \mathfrak{B}_{1}, \ldots, \mathscr{U}_{n} \mathrm{Q} \mathfrak{B}_{n}$,

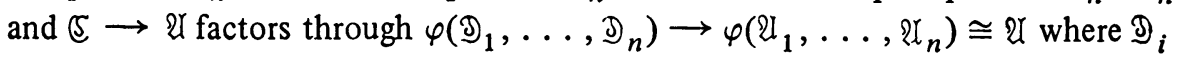
is the minimal subalgebra of $\mathfrak{Q}_{i}$. 
LemmA 7. If $\mathbf{Q}$ is a $\Phi$-decomposable relation on $\mathbf{C}$ and $\mathscr{U}$ and $B$ algebras of $\mathrm{C}$, then $\mathfrak{Q} \mathbf{Q} B$ implies $\mathfrak{O} \cong \mathfrak{B}$.

Proof. This lemma is a special case of Theorems 2 and 3 of [7].

Let $S$ be the set of quasi-logical symbols. For any $n$-place predicate symbol $R$, let " $R$ is trivial" be the sentence $\left(\forall x_{1} \cdots x_{n}\right)\left(\neg R x_{1} \cdots x_{n}\right)$ and let " $R$ is nontrivial" be its negation. For any $n$-place operation symbol $R, n \geqslant 1$, let " $R$ is trivial" be $\left(\forall x_{1} \cdots x_{n}\right)\left(R x_{1} \cdots x_{n}=x_{1}\right)$. If $R$ is a constant symbol and $a$ is a given constant of $S$, let " $R$ is trivial" be $(R=a)$. In any case, for any theory $T$ whose symbols include $R$ and those of $S, R$ is definable from $S$ in $T$ (" $R$ is trivial").

The peculiar form of the next theorem is motivated by subsequent applications. Note that for any theory whose set of symbols includes $S, T|\varnothing=T| S$.

THEOREM 8. (CONSTRUCTION THEOREM). Let $S$ be a fixed set of symbols let $\Sigma$ be a set of axioms of $S$, and let $s=\langle S, \Sigma\rangle$. Suppose that $\mathbf{Q}$ is a symmetric relation on theories and that for any theories $T$ and $T^{\prime}$ satisfying $T \mathrm{Q} T^{\prime}$, there are sets of symbols $R$ and $R^{\prime}$ which include $S$ and for which $T=T h_{s}(R)$ and $T^{\prime}=T h_{s}\left(R^{\prime}\right)$ and the following hold:

(C1). $T\left|\varnothing=T^{\prime}\right| \varnothing$.

(C2). $R_{1}, \ldots, R_{n} \in R$ implies there are $R_{1}, \ldots, R_{n} \subseteq R$ and $R_{i}^{\prime}, \ldots, R_{n}^{\prime} \subseteq R^{\prime}$ such that $R_{i} \in R_{i}, R_{i} \cap R_{j}=R_{i}^{\prime} \cap R_{j}^{\prime}=S$ for $i \neq j, R=$ $R_{1} \cup \cdots \cup R_{n}, R^{\prime}=R_{1}^{\prime} \cup \cdots \cup R_{n}^{\prime}$, and $T\left|R_{i} Q T^{\prime}\right| R_{i}^{\prime}$.

(C3). $F$ a finite subset of $R$ and $F^{\prime}$ a finite subset of $R^{\prime}$ implies $T|(R \sim F) Q T|\left(R^{\prime} \sim F^{\prime}\right)$.

(C4). $R$ and $R^{\prime}$ have no constants if $S$ has none.

(C5). If $R$ is an n-ary predicate (operation) symbol of $R$ such that no symbol of the same type occurs in $R^{\prime}$, then there are symbols $R_{1}, \ldots, R_{m}$ of $R$ of types which do occur in $R^{\prime}$ and a sentence denoted by " $R_{1}, \ldots, R_{m}$ codes an n-ary relation (operation)" in $L_{s}\left(\left\{R_{1}, \ldots, R_{m}\right\}\right)$ such that $T \mid\{R\} \cong_{s}$ $T \mid\left\{R_{1}, \ldots, R_{m}\right\} \quad\left(" R_{1}, \ldots, R_{m}\right.$ codes an $n$-ary relation (operation)").

Then for any $T$ and $T^{\prime}, T Q T^{\prime}$ implies $T \cong_{s} T^{\prime}$.

Proof. Let $S, \Sigma, s$, and $\mathbf{Q}$ be as hypothesized. Let $\mathbf{C}$ be the category whose objects are countable $\omega$-dimensional locally-finite cylindric algebras with an $S$-indexed list of constants and whose maps are homomorphisms. Note that if $R_{1}$ and $R_{2}$ are sets of symbols such that $R_{1} \cap R_{2} \subseteq S$, then $\operatorname{cyl}_{s}\left(T h_{s}\left(R_{1} \cup R_{2}\right)\right.$ ) $\cong \operatorname{cyl}_{s}\left(T h_{s}\left(R_{1}\right)\right) \otimes \operatorname{cyl}_{s}\left(T h_{s}\left(R_{2}\right)\right)$. If $T$ is a theory set of symbols includes $S$ and if $\sigma_{1}$ and $\sigma_{2}$ are sentences of $T$ 's language such that $\sigma_{1}=7 \sigma_{2}$, then $\operatorname{cyl}(T) \cong$ $\mathrm{cyl}_{s}\left(T \sigma_{1}\right) \oplus \mathrm{cyl}_{s}\left(T \sigma_{2}\right)$.

Let $\mathbf{Q}$ also denote the symmetric relation on algebras of $\mathbf{C}$ defined by $2 \mathbb{Q} B$ 
iff for some theories $T$ and $T^{\prime} \mathscr{Q}=\operatorname{cyl}_{s}(T), \mathfrak{B}=\operatorname{cyl}_{s}\left(T^{\prime}\right)$, and $T Q T^{\prime}$. We show that $\mathbf{Q}$ is $\Phi$-decomposable.

Condition (D1) is satisfied since the minimal subalgebras of related algebras $\operatorname{cyl}_{s}(T)$ and $\operatorname{cyl}_{s}\left(T^{\prime}\right)$ are $\operatorname{cyl}_{s}(T \mid \varnothing)$ and $\operatorname{cyl}_{s}\left(T^{\prime} \mid \varnothing\right)$ respectively which, by (C1), are the same.

To verify (D2), suppose $\mathscr{Y} Q \mathfrak{B}$ and $\mathfrak{E} \rightarrow \mathscr{U}$ is a monomorphism from a finitely generated algebra. Then there are theories $T$ and $T^{\prime}$ such that $\mathfrak{U}=$ $\operatorname{cyl}_{s}(T), B=\operatorname{cyl}_{s}\left(T^{\prime}\right)$, and $T Q T^{\prime}$. By the hypotheses concerning $\mathbf{Q}$, there are sets of symbols $R$ and $R^{\prime}$ such that $T=T h_{s}(R)$ and $T^{\prime}=T h_{s}\left(R^{\prime}\right)$. Since $\Subset$ is finitely generated, there is a finite set $F \subseteq R$ such that $G \rightarrow$ \& factors through the in-

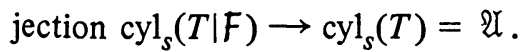

Case (i). Suppose $F=\{R\}$ for some symbol $R \in R$ whose type occurs in $R^{\prime}$. Let $R^{\prime}$ be a symbol of $R^{\prime}$ of the same type as $R$. Let $\varphi(X)=\operatorname{cyl}_{s}(T \mid\{R\})$ $\otimes X$, let $\mathfrak{X}_{1}=\operatorname{cyl}_{s}(T \mid R \sim\{R\})$, and let $\mathfrak{B}_{1}=\operatorname{cyl}_{s}\left(T^{\prime} \mid R^{\prime} \sim\left\{R^{\prime}\right\}\right)$. Then $\varphi\left(\mathscr{U}_{1}\right)$ $=\operatorname{cyl}_{s}(T \mid\{R\}) \otimes \operatorname{cyl}_{s}(T \mid R \sim\{R\}) \cong \operatorname{cyl}_{s}(T)=\mathfrak{Q} ; \varphi\left(\mathbb{B}_{1}\right)=\operatorname{cyl}_{s}(T \mid\{R\}) \otimes$ $\operatorname{cyl}_{s}\left(T^{\prime} \mid R^{\prime} \sim\left\{R^{\prime}\right\}\right) \cong$, since $T\left|\{R\}=T h_{s}(\{R\}) \cong \dddot{s}_{s} T h_{s}\left(\left\{R^{\prime}\right\}\right)=T^{\prime}\right|\left\{R^{\prime}\right\}$, $\operatorname{cyl}_{s}\left(T^{\prime} \mid\left\{R^{\prime}\right\}\right) \otimes \operatorname{cyl}_{s}\left(T^{\prime} \mid R^{\prime} \sim\left\{R^{\prime}\right\}\right) \cong \operatorname{cyl}_{s}\left(T^{\prime}\right)=\mathscr{B} ; \mathscr{U}_{1}=\operatorname{cyl}_{s}(T \mid R \sim\{R\}) \mathbf{Q}$ $\operatorname{cyl}_{s}\left(T^{\prime} \mid R^{\prime} \sim\left\{R^{\prime}\right\}\right)=\mathscr{P}_{1}$ by $(\mathrm{C} 3)$; and, noting that the minimal subalgebra $\mathscr{D}_{1}$ of $\mathfrak{P l}_{1}$ is $\operatorname{cyl}_{s}(T \mid \varnothing), \operatorname{cyl}_{s}(T \mid\{R\}) \rightarrow \operatorname{cyl}_{s}(T)=\mathfrak{Q}$ factors through $\varphi\left(\Re_{1}\right) \rightarrow \varphi\left(\mathfrak{R}_{1}\right) \cong$ थ, i.e., through $\varphi\left(\vartheta_{1}\right)=\operatorname{cyl}_{s}(T \mid\{R\}) \otimes \operatorname{cyl}_{s}(T \mid \varnothing) \rightarrow \operatorname{cyl}_{s}(T \mid\{R\}) \otimes$ $\operatorname{cyl}_{s}(T \mid R \sim\{R\}) \cong \operatorname{cyl}_{s}(T)=\mathscr{R}$.

Case (ii). Suppose $F=\{R\}$ for some $n$-ary predicate (operation) symbol $R \in R$ whose type does not occur in $R^{\prime}$. By (C5), there are symbols $R_{1}, \ldots$, $R_{m}$ in $R$ and symbols $R_{1}^{\prime}, \ldots, R_{m}^{\prime}$ in $R^{\prime}$ of corresponding type and a sentence " $R_{1}, \ldots, R_{m}$ codes an $n$-ary relation (operation)" such that $T \mid\{R\} \cong{ }_{s}$ $T \mid\left\{R_{1}, \ldots, R_{m}\right\} \quad$ (" $R_{1}, \ldots, R_{m}$ codes an $n$-ary relation (operation)") via some isomorphism $\pi$. Let " $R_{1}, \ldots, R_{m}$ codes a trivial relation (operation)" be the value $\pi$ assigns to " $R$ is trivial". Let $\sigma_{1}=$ " $R$ is nontrivial", $\sigma_{2}=$ " $R$ is trivial" $\wedge$ " $R_{1}, \ldots, R_{m}$ does not code any $n$-ary relation (operation)", and $\sigma_{3}=$ " $R$ is trivial" $\wedge$ " $R_{1}, \ldots, R_{m}$ codes an $n$-ary relation (operation)" and let $\sigma_{1}^{\prime}=$ " $R_{1}^{\prime}, \ldots, R_{m}^{\prime}$ codes an $n$-ary relation (operation)" $\wedge$ " $R_{1}^{\prime}, \ldots, R_{m}^{\prime}$ does not code a trivial relation (operation)", $\sigma_{2}^{\prime}=$ " $R_{1}^{\prime}, \ldots, R_{m}^{\prime}$ does not code an $n$-ary relation (operation)", and $\sigma_{3}^{\prime}=$ " $R_{1}^{\prime}, \ldots, R_{m}^{\prime}$ codes a trivial relation (operation)". Then $\sigma_{1}, \sigma_{2}$, and $\sigma_{3}$ are pairwise inconsistent and mutually exhaustive as are $\sigma_{1}^{\prime}$, $\sigma_{2}^{\prime}$, and $\sigma_{3}^{\prime}$. Also $T\left|\{R\}\left(\sigma_{1}\right) \cong_{s} T^{\prime}\right|\left\{R_{1}^{\prime}, \ldots, R_{m}^{\prime}\right\}\left(\sigma_{1}^{\prime}\right) ; T \mid\left\{R, R_{1}, \ldots, R_{m}\right\}\left(\sigma_{2}\right)$ $\cong_{s} T^{\prime} \mid\left\{R_{1}^{\prime}, \ldots, R_{m}^{\prime}\right\}\left(\sigma_{2}^{\prime}\right) ; T \sigma_{3} \cong_{s}$, this requires a second glance, $T \mid\left(R \sim\left\{R_{1}, \ldots, R_{m}\right\}\right)$; and $T^{\prime} \sigma_{3}^{\prime} \cong_{s} T^{\prime} \mid\left(R^{\prime} \sim\left\{R_{1}^{\prime}, \ldots, R_{m}^{\prime}\right\}\right)$. Let

$$
\begin{aligned}
\varphi\left(X_{1}, X_{2}, X_{3}\right)=\left(\operatorname{cyl}_{s}\left(T \mid\{R\} \sigma_{1}\right) \otimes X_{1}\right) & \\
& \oplus\left(\operatorname{cyl}_{s}\left(T \mid\left\{R, R_{1}, \ldots, R_{m}\right\} \sigma_{2}\right) \otimes X_{2}\right) \oplus X_{3},
\end{aligned}
$$


let $\mathscr{2}_{1}=\operatorname{cyl}_{s}(T \mid R \sim\{R\}), \Re_{2}=\operatorname{cyl}_{s}\left(T \mid R \sim\left\{R, R_{1}, \ldots, R_{m}\right\}\right)$, $\Re_{3}=$ $\operatorname{cyl}_{s}\left(T \mid R \sim\left\{R_{1}, \ldots, R_{m}\right\}\right)$, and let

$$
\mathfrak{B}_{1}=\mathfrak{B}_{2}=\mathfrak{B}_{3}=\operatorname{cyl}_{s}\left(T^{\prime} \mid R^{\prime} \sim\left\{R_{1}^{\prime}, \ldots, R_{n}^{\prime}\right\}\right) .
$$

Then

$$
\begin{aligned}
\varphi\left(\mathscr{P}_{1}, \mathscr{N}_{2}, \mathscr{N}_{3}\right)= & \left(\operatorname{cyl}_{s}\left(T \mid\{R\} \sigma_{1}\right) \otimes \operatorname{cyl}_{s}(T \mid R \sim\{R\})\right) \\
& \oplus\left(\operatorname{cyl}_{s}\left(T \mid\left\{R, R_{1}, \ldots, R_{m}\right\} \sigma_{2}\right)\right. \\
& \left.\otimes \operatorname{cyl}_{s}\left(T \mid R \sim\left\{R, R_{1}, \ldots, R_{m}\right\}\right)\right) \\
& \oplus \operatorname{cyl}_{s}\left(T \mid R \sim\left\{R_{1}, \ldots, R_{m}\right\}\right) \\
\cong & \operatorname{cyl}_{s}\left(T \sigma_{1}\right) \oplus \operatorname{cyl}_{s}\left(T \sigma_{2}\right) \oplus \operatorname{cyl}_{s}\left(T \sigma_{3}\right) \cong \operatorname{cyl}_{s}(T)=\mathfrak{R} .
\end{aligned}
$$

Similarly, $\varphi\left(\mathbb{B}_{1}, \mathfrak{B}_{2}, \mathfrak{B}_{3}\right) \cong \mathbb{B}$. By (C3), $\mathscr{N}_{1} Q \mathbb{B}_{1}, \mathbb{R}_{2} Q \mathbb{B}_{2}$, and $\mathscr{A}_{3} Q \mathfrak{B}_{3}$. Finally, the minimal subalgebras of $\mathscr{U}_{1}$, $\mathscr{U}_{2}$, and $\mathscr{U}_{3}$ are $\vartheta_{1}=\vartheta_{2}=\vartheta_{3}=\operatorname{cyl}_{s}(T \mid \varnothing)$, and $\operatorname{cyl}_{s}(T \mid\{R\}) \rightarrow \operatorname{cyl}_{s}(T)=\mathscr{U}$ factors through

which is

$$
\varphi\left(\oslash_{1}, I_{2}, \oslash_{3}\right) \rightarrow \varphi\left(\mathscr{R}_{1}, \mathscr{U}_{2}, \mathscr{U}_{3}\right)=\mathscr{U}
$$

$$
\begin{aligned}
& \left(\operatorname{cyl}_{s}\left(T \mid\{R\} \sigma_{1}\right) \otimes \operatorname{cyl}_{s}(T \mid \varnothing)\right) \\
& \quad \oplus\left(\operatorname{cyl}_{s}\left(T \mid\left\{R, R_{1}, \ldots, R_{m}\right\} \sigma_{2}\right) \otimes \operatorname{cyl}_{s}(T \mid \varnothing)\right) \\
& \quad \oplus \operatorname{cyl}_{s}(T \mid \varnothing) \rightarrow \operatorname{cyl}_{s}\left(T \mid \sigma_{1}\right) \oplus \operatorname{cyl}_{s}\left(T \mid \sigma_{2}\right) \oplus \operatorname{cyl}_{s}\left(T \mid \sigma_{3}\right) \cong \mathscr{\ell}
\end{aligned}
$$

since if $\sigma_{1}$ holds $R$ is in $\{R\}$, if $\sigma_{2}$ holds $R$ is in $\left\{R, R_{1}, \ldots, R_{m}\right\}$, and if $\sigma_{3}$ holds $R$ is trivial and hence definable from $S$.

Case (iii). Suppose $F=\left\{R_{1}, \ldots, R_{n}\right\}$. By (C2) there are $R_{1}, \ldots, R_{n} \subseteq$ $R$ and $R_{1}^{\prime}, \ldots, R_{n}^{\prime} \subseteq R^{\prime}$ such that $R_{i} \in R_{i}, R_{i} \cap R_{j}=R_{i}^{\prime} \cap R_{j}^{\prime}=S$ for $i \neq j$, $R=R_{1} \cup \cdots \cup R_{n}, R^{\prime}=R_{1}^{\prime} \cup \cdots \cup R_{n}^{\prime}$, and $T\left|R_{i} Q T^{\prime}\right| R_{i}^{\prime}$. Let $\mathscr{Q}_{i}=$ $\operatorname{cyl}_{s}\left(T \mid R_{i}\right)$ and $\mathscr{P}_{i}=\operatorname{cyl}_{s}\left(T^{\prime} \mid R_{i}^{\prime}\right)$. Then $\mathscr{R}_{i} Q \mathscr{P}_{i}$. By Cases (i) and (ii), there are $\varphi_{i} \in \Phi$, sequences $\overline{\mathcal{U}}_{i}=\left\langle\mathfrak{l}_{i}^{1}, \mathfrak{R}_{i}^{2}, \ldots\right\rangle$ and $\overline{\mathscr{P}}_{i}=\left\langle\mathscr{P}_{i}^{1}, \mathfrak{P}_{i}^{2}, \ldots\right\rangle$, and isomorphisms $\varphi_{i}\left(\overline{\mathscr{Q}}_{i}\right) \cong \mathcal{Y}_{i}$ and $\varphi_{i}\left(\overline{\mathscr{P}}_{i}\right) \cong \mathscr{P}_{i}$ such that $\mathfrak{R}_{i}^{j} \mathrm{Q} \mathscr{P}_{i}^{j}$ and $\operatorname{cyl}_{s}\left(T \mid\left\{R_{i}\right\}\right) \rightarrow$ $\operatorname{cyl}_{s}\left(T \mid R_{i}\right)=\mathscr{Q}_{i}$ factors through $\varphi_{i}\left(\overline{\mathfrak{T}}_{i}\right) \rightarrow \varphi_{i}\left(\overline{\mathscr{Q}}_{i}\right) \cong \mathscr{V}_{i}$ where $\overline{\mathfrak{D}}_{i}$ is the minimal subalgebra sequence of $\bar{\vartheta}_{i}$. Let $\varphi$ be $\varphi_{1} \otimes \varphi_{2} \otimes \cdots \otimes \varphi_{n}$, and let $\bar{\vartheta}=\bar{\vartheta}_{1} \frown$ $\overline{\mathscr{U}}_{2} \frown \cdots \frown \overline{\mathscr{U}}_{n}$ and $\overline{\mathscr{B}}=\overline{\mathscr{P}}_{1} \frown \bar{B}_{2} \frown \cdots \frown \bar{B}_{n}$ where $\frown$ is concatenation. Then $\varphi(\overline{\mathscr{V}}) \cong \varphi_{1}\left(\overline{\mathfrak{Q}}_{1}\right) \otimes \cdots \otimes \varphi_{n}\left(\overline{\mathcal{V}}_{n}\right) \cong \mathscr{V}_{1} \otimes \cdots \otimes \mathscr{N}_{n} \cong \mathfrak{U}$ and

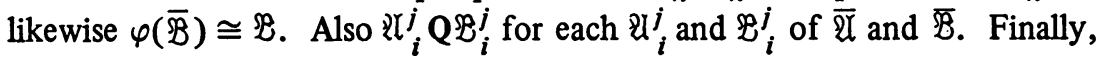
$\operatorname{cyl}_{s}\left(T \mid\left\{R_{1}, \ldots, R_{n}\right\}\right) \rightarrow \operatorname{cyl}_{s}(T)=\mathscr{U}$ factors through

$$
\begin{aligned}
\operatorname{cyl}_{s}\left(T \mid\left\{R_{1}\right\}\right) & \otimes \cdots \otimes \operatorname{cyl}_{s}\left(T \mid\left\{R_{n}\right\}\right) \\
& \rightarrow \operatorname{cyl}_{s}\left(T \mid R_{1}\right) \otimes \cdots \otimes \operatorname{cyl}_{s}\left(T \mid R_{n}\right) \cong \operatorname{cyl}_{s}(T)=\mathfrak{q}
\end{aligned}
$$


which factors through $\varphi(\overline{\mathcal{D}}) \rightarrow \varphi(\overline{\mathcal{Y}}) \cong$ Il, i.e., through

$$
\begin{aligned}
\varphi(\bar{\Im}) & =\varphi\left(\overline{\mathscr{P}}_{1}\right) \otimes \cdots \otimes \varphi_{n}\left(\overline{\mathfrak{I}}_{n}\right) \rightarrow \varphi_{1}\left(\overline{\mathscr{U}}_{1}\right) \otimes \cdots \otimes \varphi_{n}\left(\overline{\mathscr{U}}_{n}\right) \\
& \cong \mathscr{U}_{1} \otimes \cdots \otimes \mathscr{U}_{n} \cong \mathscr{U} .
\end{aligned}
$$

Hence $Q$ is $\Phi$-decomposable and by the preceding lemma $T Q T^{\prime}$ implies $T \cong T^{\prime}$

\section{Basic isomorphisms.}

LEMMA 9. For any sets $R$ and $R^{\prime}$ of symbols with no constants, if there are arbitrarily large integers such that both $R$ and $R^{\prime}$ have predicate symbols of that type or if $R$ and $R^{\prime}$ have the same unboundedness, the unboundedness is an integer, and no symbols of $R$ or $R^{\prime}$ have type larger than the unboundedness; then $\operatorname{Th}(R) \cong T h\left(R^{\prime}\right)$.

Proof. Let $Q$ be the relation such that $T Q T^{\prime}$ iff $T=T h(R)$ and $T^{\prime}=$ $T h\left(R^{\prime}\right)$ for some $R$ and $R^{\prime}$ satisfying the hypotheses of the lemma. Suppose $T=$ $T h(R)$ and $T^{\prime}=T h\left(R^{\prime}\right)$ and $T Q T^{\prime}$. Then conditions (C1)-(C4) of the Construction theorem hold. To show (C5), suppose $R \in R$ is a symbol of a type which does not occur in $R^{\prime}$. Then the hypotheses imply there is a predicate symbol $R_{0}$ $\in R$ of a type $m$ which is larger than that of $R$ and which does occur in $R^{\prime}$.

Case (i). $R$ is an $n$-ary predicate symbol. Let " $R_{0}$ codes an $n$-ary relation" be the sentence

$$
\begin{aligned}
\left(\forall x_{1} \cdots x_{n}\right) & \left(\forall x_{n+1} \cdots x_{m} x_{n+1}^{\prime} \cdots x_{m}^{\prime}\right) \\
& \left(R x_{1} \cdots x_{n} x_{n+1} \cdots x_{m} \Longleftrightarrow R x_{1} \cdots x_{n} x_{n+1}^{\prime} \cdots x_{m}^{\prime}\right) .
\end{aligned}
$$

Then $T|\{R\} \cong T|\left\{R_{0}\right\}$ (" $R_{0}$ codes an $n$-ary relation").

Case (ii). $R$ is an $n$-ary operation symbol. Let " $R_{0}$ codes an $n$-ary operation" be the sentence

$\left(\forall x_{1} \cdots x_{n}\right)(\exists y)\left(\forall x_{n+1} \cdots x_{m}\right)\left(R x_{1} \cdots x_{n} x_{n+1} \cdots x_{m} \Longleftrightarrow x_{n+1}=y\right)$.

Then $T|\{R\} \cong T|\left\{R_{0}\right\} \quad$ (" $R_{0}$ codes an $n$-ary operation").

Let $\exists \geqslant 2$ be the sentence $(\exists x y)(x \neq y)$ and let " $R$ is irreflexive" be $(\forall x)(\neg R x \cdots x)$.

LEMma 10. For any sets $R$ and $R^{\prime}$ of symbols with no constants, if there are arbitrarily large half integers such that $R$ and $R^{\prime}$ have operation symbols of that type or if $R$ and $R^{\prime}$ have the same unboundedness, the unboundedness is a half integer $\geqslant 21 / 2$, and no symbols of $R$ and $R^{\prime}$ have type larger than the unboundedness, then $\operatorname{Th}(R)(\exists \geqslant 2) \cong \operatorname{Th}\left(R^{\prime}\right)(\exists \geqslant 2)$.

Proof. Let $s=\langle\varnothing,\{\exists \geqslant 2\}\rangle$. Let $Q$ be the relation such that $T Q T^{\prime}$ iff $T=T h_{s}(R)$ and $T^{\prime}=T h_{s}\left(R^{\prime}\right)$ for some $R$ and $R^{\prime}$ satisfying the hypotheses of 
the lemma. Suppose $T=T h_{s}(R)$ and $T^{\prime}=T h_{s}\left(R^{\prime}\right)$ and $T Q T^{\prime}$. Then conditions (C1)-(C4) of the Construction theorem hold. To show (C5) suppose $R \in R$ is a symbol of a type which does not occur in $R^{\prime}$. Then the hypotheses imply there are operation symbols $R_{0}$ and $R_{1}$ in $R$ of types $m+1 / 2, m \geqslant 2$, and $k+1 / 2, k \geqslant$ 2, which are larger than that of $R$ and which do occur in $R^{\prime}$.

Case (i). $R$ is an $n$-ary operation symbol. Let " $R_{0}$ codes an $n$-ary operation" be the sentence

$$
\begin{aligned}
& \left(\forall x_{1} \cdots x_{n}\right)\left(\forall x_{n+1} \cdots x_{m} x_{n+1}^{\prime} \cdots x_{m}^{\prime}\right) \\
& \left(R x_{1} \cdots x_{n} x_{n+1} \cdots x_{m}=R x_{1} \cdots x_{n} x_{n+1}^{\prime} \cdots x_{m}^{\prime}\right) .
\end{aligned}
$$

Then $T|\{R\} \cong T|\left\{R_{0}\right\}$ (" $R_{0}$ codes an $n$-ary operation").

Case (ii). $R$ is an $n$-ary predicate symbol, $n \geqslant 1$. Let " $R_{0}$ codes an irreflexive $n$-ary relation" be " $R_{0}$ codes an $n$-ary operation" $\wedge$

$$
\begin{aligned}
& \left(\forall x_{1} \cdots x_{n} x_{n+1} \cdots x_{m}\right) \\
& \left(R x_{1} \cdots x_{m}=x_{1} \vee{ }_{1 \leqslant i \leqslant n}\left(x_{1}=x_{2}=\cdots=x_{i}\right.\right. \\
& \left.\left.\quad \neq x_{i+1} \wedge R x_{1} \cdots x_{m}=x_{i+1}\right)\right),
\end{aligned}
$$

and let " $R_{1}$ codes a unary relation" be

$\left(\forall x_{1}\right)\left[\left(\forall x_{2} \cdots x_{k}\left(R x_{1} \cdots x_{k}=x_{1}\right)\right) \vee\left(\forall x_{2} \cdots x_{k}\left(R x_{1} \cdots x_{k}=x_{2}\right)\right)\right]$.

Then for any unary predicate symbol $U \notin R, T|\{R\} \cong T|\{R\}$ (" $R$ is irreflexive") " $\otimes$ " $(T \mid(R \sim\{R\}) \cup\{U\}) \cong T \mid\left\{R_{0}, R_{1}\right\} \quad$ (" $R_{0}$ codes an irreflexive $n$ ary relation" $\wedge$ " $R_{1}$ codes a unary relation").

Case (iii). $R$ is a 0 -ary predicate. Let " $R_{0}$ codes a 0 -ary relation" be $\left(\forall x_{1} \cdots x_{m}\left(R x_{1} \cdots x_{m}=x_{1}\right)\right) \vee\left(\forall x_{1} \cdots x_{m}\left(R x_{1} \cdots x_{m}=x_{2}\right)\right)$. Then $T|\{R\} \cong T|\left\{R_{0}\right\}$ (" $R_{0}$ codes a 0 -ary predicate").

LEMMA 11. For any sets of symbols $R$ and $R^{\prime}$ with at least one constant and infinitely many symbols of type $\geqslant 1$, if $R$ and $R^{\prime}$ have exactly the same number of symbols of each type other than $1 / 2$, then $T h(R) \cong T h\left(R^{\prime}\right)$.

Proof. We may assume without loss of generality that $R$ and $R^{\prime}$ contain a common constant $a$. Let $s=\langle\{a\}, \varnothing\rangle$. Let $Q$ be the relation such that $T Q T^{\prime}$ iff $T=T h_{s}(R)$ and $T=T h_{s}\left(R^{\prime}\right)$ for some $R$ and $R^{\prime}$ satisfying the hypotheses of the lemma and the assumption above. Suppose $T=T h_{s}(R)$ and $T^{\prime}=T h_{s}\left(R^{\prime}\right)$ and $T \mathrm{Q} T^{\prime}$. Then conditions (C1) $-(\mathrm{C} 4)$ of the Construction theorem hold. To show (C5) suppose $R \in R$ is a symbol of a type which does not occur in $R^{\prime}$. Then the hypotheses imply $R$ is some constant $b$ and there is a predicate or operation symbol $R_{0}$ of $R$ of a type $m$ or $m+1 / 2, m \geqslant 1$, which also occurs in $R^{\prime}$.

If $R_{0}$ is a predicate symbol, let " $R_{0}$ codes a constant" be $(\exists y)\left(\forall x_{1} \cdots\right.$ $\left.x_{m}\right)\left(R x_{1} \cdots x_{m} \Longleftrightarrow x_{1}=y\right)$. If $R_{0}$ is an operation symbol, let " $R_{0}$ codes a 
constant" be $(\exists y)\left(\forall x_{1} \cdots x_{m}\right)\left(R x_{1} \cdots x_{m}=y\right)$. Then $T\left|\{b\} \cong_{s} T\right|\left\{R_{0}\right\}$ (" $R_{0}$ codes a constant").

LEMMA 12. For any set of symbols $R$ and $R^{\prime}$ with at least one constant and infinitely many symbols of type $\geqslant 1$, if $R$ and $R^{\prime}$ have exactly the same number of symbols of each type other than 0 then $\operatorname{Th}(R)(\exists \geqslant 2) \cong \operatorname{Th}\left(R^{\prime}\right)(\exists \geqslant 2)$.

Proof. We may assume that $R$ and $R^{\prime}$ contain a common constant $a$. Let $s=\langle\{a\},\{\exists \geqslant 2\}\rangle$. Let $Q$ be the relation such that $T Q T^{\prime}$ iff $T=T h_{s}(R)$ and $T^{\prime}=T h_{s}\left(R^{\prime}\right)$ for some $R$ and $R^{\prime}$ satisfying the hypotheses of the lemma and the assumption above. Suppose $T=T h_{s}(R)$ and $T^{\prime}=T h_{s}\left(R^{\prime}\right)$ and $T Q T^{\prime}$. Then conditions (C1)-(C4) of the Construction theorem hold. To show (C5) suppose $R \in R$ is a symbol of a type which does not occur in $R^{\prime}$. Then the hypotheses imply $R$ is some 0 -ary predicate $P$ and there is a predicate or operation symbol $R_{0}$ of $R$ of a type $m$ or $m+1 / 2, m \geqslant 1$, which also occurs in $R^{\prime}$.

If $R_{0}$ is a predicate symbol, let " $R_{0}$ codes a 0 -ary predicate" be $\left(\forall x_{1} \cdots\right.$ $\left.x_{m}\left(R_{0} x_{1} \cdots x_{m}\right)\right) \vee\left(\forall x_{1} \cdots x_{m}\left(7 R_{0} x_{1} \cdots x_{m}\right)\right)$. If $R_{0}$ is an operation symbol, let " $R_{0}$ codes a 0 -ary predicate" be $\left(\forall x_{1} \cdots x_{m}\left(R_{0} x_{1} \cdots x_{m}=x_{1}\right)\right)$ $\vee\left(\forall x_{1} \cdots x_{m}\left(R_{0} x_{1} \cdots x_{m}=a\right)\right)$. Then $T\left|\{P\} \cong_{s} T\right|\left\{R_{0}\right\} \quad$ (" $R_{0}$ codes a 0 -ary predicate").

LEMMA 13. For any sets of symbols $R$ and $R^{\prime}$ with at least one constant, infinitely many symbols of type $\geqslant 1$, and infinitely many of type 0 , if $R$ and $R^{\prime}$ have exactly the same number of symbols of each type other than 1 , then $\operatorname{Th}(R)(\exists \geqslant 2) \cong \operatorname{Th}\left(R^{\prime}\right)(\exists \geqslant 2)$.

Proof. We may assume without loss of generality that $R$ and $R^{\prime}$ contain a common constant $a$. Let $s=\langle\{a\},\{\exists \geqslant 2\}\rangle$. Let $\mathbf{Q}$ be the relation such that $T Q T^{\prime}$ iff $T=T h_{s}(R)$ and $T^{\prime}=T h_{s}\left(R^{\prime}\right)$ for some $R$ and $R^{\prime}$ satisfying the hypotheses of the lemma and the assumption above. Suppose $T=T h_{s}(R)$ and $T^{\prime}=T h_{s}\left(R^{\prime}\right)$ and $T Q T^{\prime}$. Then conditions (C1)-(C4) of the Construction theorem hold. To show (C5) suppose $R \in R$ is a symbol of a type which does not occur in $R^{\prime}$. Then the hypotheses imply $R$ is some unary predicate $U$ and there is 0 -ary predicate symbol $P \in R$ and a predicate or operation symbol $R_{0} \in R$ of a type $m$ or $m+1 / 2, m \geqslant 1$ which also occurs in $R^{\prime}$.

If $R_{0}$ is a predicate symbol, let " $R_{0}, P$ codes a unary predicate" be

$$
\left(\forall x_{1}\right)\left(\forall x_{2} \cdots x_{m} x_{2}^{\prime} \cdots x_{m}^{\prime}\right)\left(R x_{1} x_{2} \cdots x_{m} \Longleftrightarrow R x_{1} x_{2}^{\prime} \cdots x_{m}^{\prime}\right) .
$$

If $R_{0}$ is an operation symbol, let " $R_{0}, P$ codes a unary predicate" be $\left(\forall x_{1} \cdots\right.$ $\left.x_{m}\right)\left(R x_{1} \cdots x_{m}=x_{1} \vee R x_{1} \cdots x_{m}=a\right)$. The idea is that $x \in U$ is determined by $R_{0}$ for $x \neq a$ and by $P$ for $x=a$. Then $T\left|\{U\} \cong_{s} T\right|\left\{R_{0}, P\right\}$ (" $R_{0}$, $P$ codes a unary predicate'). 
Let $\exists ! 1$ be the sentence $(\forall x y)(x=y)$.

LEMma 14. For any sets $R$ and $R^{\prime}$ of symbols, if $R$ and $R^{\prime}$ have the same number of predicate symbols, then $\operatorname{Th}(R)(\exists ! 1) \cong \operatorname{Th}\left(R^{\prime}\right)(\exists ! 1)$.

Proof. On a set with exactly one element, an operation symbol has exactly one possible interpretation and a predicate symbol exactly two. Thus all operation symbols are definable and eliminable and all predicate symbols can be replaced by 0 -ary predicates. Hence the lemma.

Classification theorem.

TheOREM 15 (ClassificAtion THEOREM). The following properties and functions are a complete set of invariants for countable languages under cylindric isomorphisms.

(1) The reduced type sequence.

(2) The existence of a constant.

(3) The number of predicate symbols.

(4) The number of unary predicate symbols in case the language's only other symbols are for 0-ary predicates and unary operations.

Proof. The four properties have been shown to be invariants. Suppose $T$ and $T^{\prime}$ are the theories of two languages $L$ and $L^{\prime}$ with symbols $R$ and $R^{\prime}$ which are the same with respect to the invariants. We must show that $T$ and $T^{\prime}$ are cylindrically isomorphic.

Since $L$ and $L^{\prime}$ have the same reduced type sequence, they have the same unboundedness. If the unboundedness is undefined, $R$ and $R^{\prime}$ are finite and so the reduced type sequence is the full type sequence and hence $T \cong T^{\prime}$. Suppose the unboundedness is defined. Let $R_{0}$ and $R_{0}^{\prime}$ be the set of symbols of $R$ and $R^{\prime}$ respectively whose type is less than or equal to the unboundedness. Then $R \sim$ $R_{0}$ and $R^{\prime} \sim R_{0}^{\prime}$ are finite and have the same type sequence. Hence $T \mid\left(R \sim R_{0}\right)$ $\cong T^{\prime} \mid\left(R^{\prime} \sim R_{0}^{\prime}\right)$. Thus to show $T \cong T^{\prime}$ it suffices to show $T\left|R_{0}^{\prime} \cong T^{\prime}\right| R_{0}^{\prime}$. Consequently, we may henceforth assume $R$ and $R^{\prime}$ have no symbols of type greater than the unboundedness.

If the unboundedness is 0 , the reduced type sequence is the full sequence and hence $T \cong T^{\prime}$. If the unboundedness is $1 / 2$, the reduced type sequence and the number of predicate symbols determine the full type sequence and hence $T \cong T^{\prime}$. Henceforth, assume the unboundedness of $R$ and $R^{\prime}$ is $\geqslant 1$.

Suppose $R$ and $R^{\prime}$ have constants $a$ and $a^{\prime}$ respectively Let $R_{0}$ and $R_{0}^{\prime}$ be $R$ and $R^{\prime}$ respectively minus all constants except $a$ and $a^{\prime}$. By Lemma $11, T \cong T \mid R_{0}$ and $T^{\prime} \cong T^{\prime} \mid R_{0}^{\prime}$. Hence to show $T \cong T^{\prime}$, it suffices to show $T\left|R_{0} \cong T^{\prime}\right| R_{0}^{\prime}$. Henceforth assume $R$ and $R^{\prime}$ have no constants or exactly one constant each, $a$ and $a^{\prime}$ respectively. 
Since $R$ and $R^{\prime}$ have the same number of predicate symbols, $T(\exists ! 1) \cong$ $T^{\prime}(\exists ! 1)$ by Lemma 14 . It remains to be shown that $T(\exists \geqslant 2) \cong T^{\prime}(\exists \geqslant 2)$.

Suppose the unboundedness is $\neq 1 \frac{1 / 2}{2}$ and $\neq \omega$. If there are no constants, then $T(\exists \geqslant 2) \cong T^{\prime}(\exists \geqslant 2)$ by Lemma 9 or 10 . If there are unique constants $a$ and $a^{\prime}$, then $T(\exists \geqslant 2)\left|\{a\} \cong T^{\prime}(\exists \geqslant 2)\right|\left\{a^{\prime}\right\}$ and, by Lemma 9 or 10 , $T(\exists \geqslant 2)|(R \sim\{a\}) \cong T(\exists \geqslant 2)|\left(R^{\prime} \sim\left\{a^{\prime}\right\}\right)$. Hence $T(\exists \geqslant 2) \cong T^{\prime}(\exists \geqslant 2)$.

Suppose the unboundedness is $\omega$. Then, in the case of no constants, $T(\exists \geqslant 2)=T h(R)(\exists \geqslant 2) \cong$, by Lemma 9 or $10, \operatorname{Th}\left(R \cup R^{\prime}\right)(\exists \geqslant 2) \cong$ $\operatorname{Th}\left(R^{\prime}\right)(\exists \geqslant 2)=T^{\prime}(\exists \geqslant 2)$. The case with a unique constant is handled similarly as in the previous paragraph.

Suppose the unboundedness is $1 \frac{1 / 2}{2}$ and there are no constants. If the number of unary predicates in $R$ and/or $R^{\prime}$ is finite, then the reduced type sequence, the number of predicate symbols, and the number of unary predicate symbols determine the full type sequence and hence $T(\exists \geqslant 2) \cong T^{\prime}(\exists \geqslant 2)$. If there are infinitely many unary predicates, let $R_{0}$ and $R_{0}^{\prime}$ be the set of unary operation symbols of $R$ and $R^{\prime}$ respectively. Then $T(\exists \geqslant 2)\left|R_{0} \cong T^{\prime}(\exists \geqslant 2)\right| R_{0}^{\prime}$ is clear and $T(\exists \geqslant 2)\left|\left(R \sim R_{0}\right) \cong T^{\prime}(\exists \geqslant 2)\right|\left(R^{\prime} \sim R_{0}^{\prime}\right)$ follows from Lemma 9. Hence $T(\exists \geqslant 2) \cong T^{\prime}(\exists \geqslant 2)$.

Suppose the unboundedness is $1 \frac{112}{2}$ and there are unique constants $a$ and $a^{\prime}$. Let $U$ be a countably infinite set of propositional letters disjoint from $R$ and $R^{\prime}$. Then $T(\exists \geqslant 2)=\operatorname{Th}(R)(\exists \geqslant 2) \cong$, by Lemma $12, \operatorname{Th}(R \cup U)(\exists \geqslant 2) \cong$, by Lemma $13, \operatorname{Th}\left(R^{\prime} \cup U\right)(\exists \geqslant 2) \cong \operatorname{Th}\left(R^{\prime}\right)(\exists \geqslant 2)=T(\exists \geqslant 2)$.

Hence $T \cong T^{\prime}$.

While we have considered only countable languages, the generalization of the Classification theorem to languages of arbitrary cardinality is not too difficult.

The corresponding classification problem for the Boolean algebras of sentences of countable languages has been solved by Hanf and his student, Simons. In this case all languages of infinite similarity type have isomorphic Boolean algebras. So do all languages of finite similarity type with at least one symbol of type $\geqslant 2$ or at least two symbols of type $1 \frac{1}{2}$. So do all languages of finite similarity type with no symbols of type $\geqslant 2$ and exactly one of type $1 \frac{1}{2}$. The remaining cases are all nonisomorphic.

\section{REFERENCES}

1. Karel de Bouvère, Synonymous theories, Theory of Models (Proc. 1963 Internat. Sympos. Berkeley), North-Holland, Amsterdam, 1965.

2. Stephen D. Comer, $A$ sheaf-theoretic duality theory for cylindric algebras, Trans. Amer. Math. Soc. 169 (1972), 75-87. MR 46 \#7023.

3. William Hanf, Primitive Boolean algebras, Proc. Sympos. Pure Math., vol. 25, Amer. Math. Soc., Providence, R. I., 1974, pp. 75-90.

4. L. Henkin, J. D. Monk and A. Tarski, Cylindric algebras. Part I. With an introductory chapter: General theory of algebras, Studies in Logic and the Foundations of Math., vol. 
64, North-Holland, Amsterdam and London, 1971. MR 47 \#3171.

5. Simon Kochen, Ultraproducts in the theory of models, Ann. of Math. (2) 74 (1961), 221-261. MR 25 \#1992.

6. Richard Montague, Contributions to the axiomatic foundations of set theory, Ph. D. Thesis, Univ. of California, Berkeley, Calif., 1957.

7. Dale Myers, The back-and-forth isomorphism construction, Pacific J. Math. 55 (1974), 521-529.

8. Abraham Robinson, Introduction to model theory and to the metamathematics of algebras, North-Holland, Amsterdam, 1963. MR 27 \#3533.

9. Saharon Shelah, Every two elementary equivalent models have isomorphic ultrapowers, Israel J. Math. 10 (1971), 224-233. MR 45 \#6608.

10. Roger Simons, The Boolean algebra of sentences of the theory of a function, $\mathrm{Ph}$. D. Thesis, Univ. of California, Berkeley, Calif. 1972.

DEPARTMENT OF MATHEMATICS, UNIVERSITY OF HAWAII, HONOLULU, HAWAII 96822 